\title{
Patterns and risks of postoperative recurrence in completely resected EGFR-mutant non-small cell lung cancer: prognostic significance of routine immunohistochemical markers
}

\author{
Jianjiao $\mathrm{Ni}^{1,2,3 \#}$, Tiantian Guo ${ }^{1,2,3 \#}$, Yuan $\mathrm{Li}^{2,3,4}, \mathrm{Xi}$ Yang ${ }^{1,2,3}$, Yida $\mathrm{Li}^{1,2,3}$, Liqing Zou ${ }^{1,2,3}$, Li Chu ${ }^{1,2,3}, \mathrm{Xiao}^{1,2,3}$ \\ $\mathrm{Chu}^{1,2,3}$, Shuyan $\mathrm{Li}^{1,2,3}$, Luxi Ye ${ }^{1,2,3}$, Yawei Zhang ${ }^{2,3,5}$, Zhengfei Zhu ${ }^{1,2,3}$ \\ ${ }^{1}$ Department of Radiation Oncology, Fudan University Shanghai Cancer Center, Shanghai 200032, China; ${ }^{2}$ Department of Oncology, Shanghai \\ Medical College, ${ }^{3}$ Institute of Thoracic Oncology, Fudan University, Shanghai 200032, China; ${ }^{4}$ Department of Pathology, ${ }^{5}$ Department of Thoracic \\ Surgery, Fudan University Shanghai Cancer Center, Shanghai 200032, China \\ Contributions: (I) Conception and design: J Ni, T Guo, Z Zhu; (II) Administrative support: None; (III) Provision of study materials or patients: Y Li, \\ Y Zhang, Z Zhu; (IV) Collection and assembly of data: T Guo, Y Li, X Yang, Y Li, L Zou; (V) Data analysis and interpretation: J Ni, L Chu, X Chu, \\ S Li, L Ye; (VI) Manuscript writing: All authors; (VII) Final approval of manuscript: All authors. \\ \#These authors contributed equally to this work. \\ Correspondence to: Zhengfei Zhu, MD. Department of Radiation Oncology, Fudan University Shanghai Cancer Center, 270 Dong An Road, Shanghai \\ 200032, China. Email: fuscczzf@163.com.
}

Background: Recent studies indicate that EGFR-mutant non-small cell lung cancer (NSCLC) is a heterogeneous disease with varying prognosis. In order to design an optimized surveillance strategy and identify potential candidates for adjuvant therapy, the patterns and risks of postoperative recurrence in completely resected EGFR-mutant NSCLC should be investigated, which are currently largely unknown.

Methods: Consecutive patients with curatively resected EGFR-positive NSCLC receiving standard adjuvant chemotherapy without EGFR tyrosine kinase inhibitors (TKI), with or without adjuvant radiotherapy, from January 2007 to December 2017 in our cancer center, were retrospectively reviewed. Prognostic significance of ten routine immunohistochemical (IHC) markers were examined.

Results: After a median follow-up of 32 (range, 5-122) months, disease recurrence occurred in 197 (37.1\%) of the 531 enrolled patients. The frequencies of thoracic recurrence, brain recurrence, bone recurrence, abdominal recurrence and neck recurrence, were $69.0 \%, 20.8 \%, 20.8 \%, 7.1 \%$ and $6.6 \%$, respectively. Using the Cox regression model, tumor size, Ki67, CK20, and N stage were identified as independent predictors of overall recurrence. A nomogram predicting the 1-, 2-, and 3-year cumulative rate of overall recurrence was then developed and internally validated, with a bias-corrected C-index of 0.723 (95\% CI, 0.675 to 0.771 ) and a small extent of "over-fitting" (0.8\%). Risk factors of site-specific recurrence were also discovered. Additionally, using competing risk analyses, $\mathrm{N}$ stage, lymphovascular invasion (LVI) and CK5/6 were found as independent predictors of loco-regional recurrence. Among patients with N2-positive disease ( $\mathrm{n}=91$ ), adjuvant radiotherapy tended to prolong disease free survival (DFS) $(\mathrm{P}=0.067)$, but not overall survival (OS) $(\mathrm{P}=0.271)$.

Conclusions: This study provides the proof of concept of using routine IHC markers, along with common clinical-pathological parameters, in predicting postoperative recurrence among completely resected EGFRmutant NSCLC. Adjuvant radiotherapy may improve DFS, but hard to prolong OS in N2-positive EGFRmutant NSCLC without further biomarker-guided patients' selection.

Keywords: Non-small cell lung cancer (NSCLC); EGFR mutation; recurrence; immunohistochemical markers; adjuvant therapy

Submitted Aug 16, 2019. Accepted for publication Nov 13, 2019.

doi: $10.21037 /$ tlcr.2019.12.02

View this article at: http://dx.doi.org/10.21037/tlcr.2019.12.02 


\section{Introduction}

Non-small cell lung cancer (NSCLC) is the predominant subtype of lung cancer (1) and surgery remains to be the first treatment choice for early-stage NSCLC (2). However, postoperative recurrence still poses a significant threat to long-term survival and patients' quality of life (3). Cisplatin-based adjuvant chemotherapy is the standard of care among patients with resected stage II-III NSCLC as well as a subset of stage IB NSCLC with extra risk factors, resulting in a 5 -year disease free survival (DFS) lower than $30 \%$, at the cost of considerable toxicity (4). Additionally, accumulating evidence suggests that adjuvant radiotherapy could further improve DFS for N2-positive patients, but controversies remain $(5,6)$.

EGFR mutations can be detected in about $15 \%$ and up to $50 \%$ of NSCLC, among Caucasian and Asian populations, respectively (7). EGFR-mutant NSCLC defines a distinct molecular subtype of lung cancers, which is sensitive to EGFR tyrosine kinase inhibitors (TKIs) (8). Hence, there are studies supporting the administration of EGFR TKIs in the adjuvant setting for patients with EGFR-mutant NSCLC (9-12). On the other hand, recent studies indicate that EGFR-mutant NSCLC is a heterogeneous disease with varying co-mutations, different histologic subtypes and distinct expression of oncoproteins (13-15). And thus, in order to design the optimal treatment strategy for adjuvant therapy, the patterns and risks of postoperative recurrence in curatively resected EGFR-positive NSCLC should be investigated.

Recently, various routine immunohistochemical (IHC) markers have been found to have significant prognostic values in different human cancers, including esophageal adenocarcinoma (16), esophageal squamous cell carcinoma (17), urothelial carcinoma (18), and breast cancer (19). IHC analyses of Ki67, Her-2, TTF1, CK7, CK5/6, CK20, P63, synaptophysin (Syn), RRM1, and NapsinA are routinely performed for resected NSCLC, for the help of disease diagnosis and differential diagnosis (20). Nevertheless, few studies concerning the prognostic significance of these routinely used IHC markers in curatively resected EGFR-mutant NSCLC, are reported.

\section{Methods}

\section{Patients and clinical data collection}

We retrospectively reviewed NSCLC patients who underwent surgical resection with curative intention and had their postoperative samples tested for EGFR mutations at Fudan University Shanghai Cancer Center from January 2007 to December 2017. Patients who underwent complete surgical resection (21), had pathologically confirmed EGFR mutation-positive stage I-III NSCLC and received standard adjuvant chemotherapy in comply with NCCN guidelines (22), were enrolled in our study. Of note, patients who received adjuvant radiotherapy and those who did not, were both allowed to be included. Exclusion criteria included neoadjuvant therapy, a second primary tumor, compromised resection, positive surgical margins, and death due to surgical complications.

Clinical-pathological characteristics of each patient were obtained from electronic medical records including age, sex, smoking status, tumor size, TNM stage, tumor differentiation, tumor histology, lymphovascular invasion, visceral pleural invasion, the Eastern Corporative Oncology Group (ECOG) performance score and EGFR mutation subtype. Age at the time of curative resection was classified into two groups ( $\leq 65$ and $>65$ years). Pathologic TNM stage was in accordance with the eighth edition Lung Cancer Stage Classification (23). Tumor differentiation was determined on the basis of the 2015 World Health Organization Classification of Tumors of the Lung, Pleura, Thymus and Heart (24). Histology of adenocarcinoma was categorized using the International Association for the Study of Lung Cancer, American Thoracic Society, and European Respiratory Society (IASLC/ATS/ERS) lung adenocarcinoma multidisciplinary classification (25).

Detection of EGFR mutations was performed using the AMRS (amplification-refractory mutation system) method, and EGFR mutation subtypes were divided into two groups: common mutations including L858R and exon 19 deletion, and uncommon mutations such as G719X, L861Q, S768I and exon 20 insertions. IHC staining and evaluation were routinely performed in the Immunohistochemistry Diagnostic Laboratory of our cancer center. Data on the expression status of Her-2, TTF1, CK20, CK5/6, CK7, P63, RRM1, NapsinA, Ki67, and Syn, were collected for each patient.

\section{Follow-up and postoperative recurrence}

Follow-up was scheduled at regular intervals: every 3 months after surgery in the first 3 years, every 6 months for 3-5 years, and every 12 months afterward. Chest computed tomography (CT) scans and ultrasonography of abdominal and cervical regions were routinely performed, 
while brain magnetic resonance imaging (MRI) and bone scanning were not mandatory, but at the discretion of the treating physicians. Telephone follow-up calls were also implemented.

Postoperative recurrence was diagnosed considering all the evidence provided by CT, MRI, positron emission tomography-computed tomography (PET-CT) scan or pathologic confirmation. Site-specific recurrence was categorized into five groups: thoracic recurrence, brain recurrence, neck recurrence, abdominal recurrence, and bone recurrence. Metastasis to the region from the diaphragm to the pelvis as the initial recurrence site was described as abdominal recurrence. Neck recurrence indicated the initial recurrence in the cervical region, mainly compromising the cervical lymph node metastasis. The first relapse in the chest wall and thoracic cavity was defined as thoracic recurrence. Furthermore, initial recurrence developed at the resection margin, the anastomosis or the mediastinal or ipsilateral hilar lymph nodes, were considered as loco-regional recurrence. All other failures were defined as distant recurrence. Of note, thoracic recurrences that displayed as thoracic metastases or chest well metastases, which could not be categorized as loco-regional recurrence, were also defined as distant recurrence.

Recurrence free survival (RFS) was calculated from the date of pathological diagnosis to the documentation of sitespecific recurrence or overall recurrence. Patients were censored at the last time of follow-up when no evidence of recurrence was found. Patients who died without recurrence were censored at time of death. DFS was calculated from the date of pathological diagnosis to the documentation of tumor recurrence or death due to any causes. Overall survival (OS) was calculated from the date of pathological diagnosis to death due to any causes. Our study followed The Declaration of Helsinki. The institutional review board of Fudan University Shanghai Cancer Center approved the study (IRB\#090977-1). Informed consent was waived by the institutional review board because this was a retrospective study.

\section{Statistical analysis}

The indicators of overall recurrence were identified using the Cox proportional hazard regression model. The predictors of loco-regional recurrence and site-specific recurrence were selected using competing risk methodology and Stata version 13.1 software (StataCorp, College Station, TX, USA). The hazard ratio (HR) and the $95 \%$ confidence interval (CI) were calculated using coefficients from the model. The "rms" package in $\mathrm{R}$ project was used to construct a nomogram with independent variables selected in multivariate analysis. The Harrell's concordance index (C-index) was calculated, which represented the model's discriminative ability. For internal validation, the bootstrapping method (one thousand repetitions) was applied to evaluate the bias-corrected C-index and the extent of "over fitting" (26). For the evaluation of predictive accuracy, the calibration curves were plotted to compare nomogram-predicted and actual observed RFS probability. Kaplan-Meier method was used to estimate survival, and differences among groups were investigated by the logrank test. Statistical analysis was performed using SPSS 21.0 (SPSS, Chicago, IL, USA) and R version 3.5.1 (The R Foundation for Statistical Computing). All assessment is considered to be significant when two-sided $\mathrm{P}$ value is less than 0.05 .

\section{Results}

\section{Patient characteristics and expression of IHC markers}

A total of 531 patients were finally enrolled in this study and a flowchart for patient selection in this study was presented in Figure S1. Of note, the baseline disease characteristics (including age, sex, TNM stage and tumor histology) of the 1,183 patients tested for EGFR mutations and the whole population who underwent curative resection of NSCLC during the same time period, were generally well balanced (Table S1). Detailed baseline disease characteristics and expression levels of each IHC markers of the enrolled patients, were illustrated in Table 1.

\section{Patterns of postoperative recurrence}

Post-surgery, 169 patients received adjuvant chemotherapy (including all of the 158 patients with stage II-III NSCLC and 11 of the 109 patients with stage IB NSCLC and extra risk factors) and 28 patients received adjuvant radiotherapy (all of the patients had N2-positive NSCLC). After a median follow-up of 32 months (range, 5-122 months), disease recurrence was observed in 197 patients and 4 patients died without documentation of disease recurrence. The median time from curative surgery to initial recurrence was 19 (95\% CI, 16.63-21.37) months and the patterns of postoperative recurrence were displayed in Figure 1A. Briefly, $136(69.0 \%)$ patients had thoracic recurrence, $41(20.8 \%)$ had brain recurrence, 41 (20.8\%) had bone recurrence, 
Table 1 Disease characteristics and expression of immunohistochemical markers

\begin{tabular}{|c|c|}
\hline Clinical-pathological characteristics & Number of patients (\%) \\
\hline \multicolumn{2}{|l|}{ Age (years) } \\
\hline$\leq 65$ & $409(77.0)$ \\
\hline$>65$ & $122(23.0)$ \\
\hline \multicolumn{2}{|l|}{ Sex } \\
\hline Female & $325(61.2)$ \\
\hline Male & $206(38.8)$ \\
\hline \multicolumn{2}{|l|}{ Smoking status } \\
\hline Never & $418(78.7)$ \\
\hline Ever & $113(21.3)$ \\
\hline \multicolumn{2}{|l|}{ Tumor size $(\mathrm{cm})$} \\
\hline$\leq 2.0$ & $271(51.0)$ \\
\hline$>2.0$ & $260(49.0)$ \\
\hline \multicolumn{2}{|l|}{ T stage } \\
\hline T1 & $404(76.1)$ \\
\hline $\mathrm{T} 2$ & $105(19.8)$ \\
\hline T3 & $14(2.6)$ \\
\hline $\mathrm{T} 4$ & $8(1.5)$ \\
\hline \multicolumn{2}{|l|}{ N stage } \\
\hline No & $388(73.1)$ \\
\hline $\mathrm{N} 1$ & $52(9.8)$ \\
\hline $\mathrm{N} 2$ & $91(17.1)$ \\
\hline \multicolumn{2}{|l|}{ Pathologic TNM stage } \\
\hline I & $373(70.2)$ \\
\hline II & $61(11.5)$ \\
\hline III & $97(18.3)$ \\
\hline \multicolumn{2}{|l|}{ ECOG performance score } \\
\hline $0-1$ & $504(94.9)$ \\
\hline 2 & $27(5.1)$ \\
\hline \multicolumn{2}{|l|}{ Type of EGFR mutation } \\
\hline L858R & $228(42.9)$ \\
\hline 19del & $244(46.0)$ \\
\hline Uncommon mutations & $59(11.1)$ \\
\hline \multicolumn{2}{|l|}{ Lymphovascular invasion } \\
\hline Absent & $434(81.7)$ \\
\hline Present & $97(18.3)$ \\
\hline
\end{tabular}

Table 1 (continued)

\begin{tabular}{|c|c|}
\hline Clinical-pathological characteristics & Number of patients (\%) \\
\hline \multicolumn{2}{|l|}{ Visceral pleural invasion } \\
\hline Absent & $436(82.1)$ \\
\hline Present & 95 (17.9) \\
\hline \multicolumn{2}{|l|}{ Histological differentiation } \\
\hline Well & $56(10.5)$ \\
\hline Moderate & $441(83.1)$ \\
\hline Poor & $34(6.4)$ \\
\hline \multicolumn{2}{|l|}{ Histology } \\
\hline \multicolumn{2}{|l|}{ Adenocarcinoma } \\
\hline Lepidic & $23(4.3)$ \\
\hline Acinar & 366 (68.9) \\
\hline Papillary & $73(13.8)$ \\
\hline Micropapillary & $16(3.0)$ \\
\hline Solid & $38(7.2)$ \\
\hline Variant & $7(1.3)$ \\
\hline Adenosquamous & $8(1.5)$ \\
\hline \multicolumn{2}{|l|}{ Her2 } \\
\hline- & $314(59.1)$ \\
\hline+ & 217 (40.9) \\
\hline \multicolumn{2}{|l|}{ Ki67 } \\
\hline$\leq 10 \%$ & $276(52.0)$ \\
\hline$>10 \%$ & $255(48.0)$ \\
\hline \multicolumn{2}{|l|}{ TTF1 } \\
\hline- & $59(11.2)$ \\
\hline+ & $472(88.8)$ \\
\hline \multicolumn{2}{|l|}{ CK20 } \\
\hline- & $494(93.0)$ \\
\hline+ & $37(7.0)$ \\
\hline \multicolumn{2}{|l|}{ CK7 } \\
\hline- & $463(87.2)$ \\
\hline+ & 68 (12.8) \\
\hline \multicolumn{2}{|l|}{ CK5/6 } \\
\hline- & $503(94.7)$ \\
\hline+ & 28 (5.3) \\
\hline
\end{tabular}

Table 1 (continued)

Table 1 (continued) 
Table 1 (continued)

\begin{tabular}{lc}
\hline Clinical-pathological characteristics & Number of patients (\%) \\
\hline P63 & $401(75.5)$ \\
- & $130(24.5)$ \\
+ & \\
RRM1 & $506(95.3)$ \\
- & $25(4.7)$ \\
+ & \\
NapsinA & $50(9.4)$ \\
- & $481(90.6)$ \\
+ & \\
Syn & $463(87.2)$ \\
- & $68(12.8)$ \\
+ &
\end{tabular}

EGFR, epidermal growth factor receptor; ECOG, the Eastern Corporative Oncology Group.

\section{A}

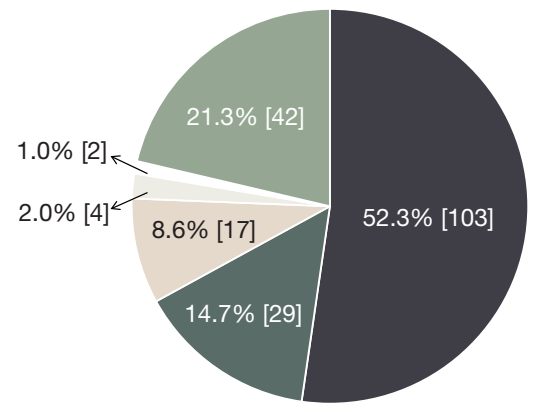

-Thorax -Brain Bone Abdomen Neck $=$ Multiple sites

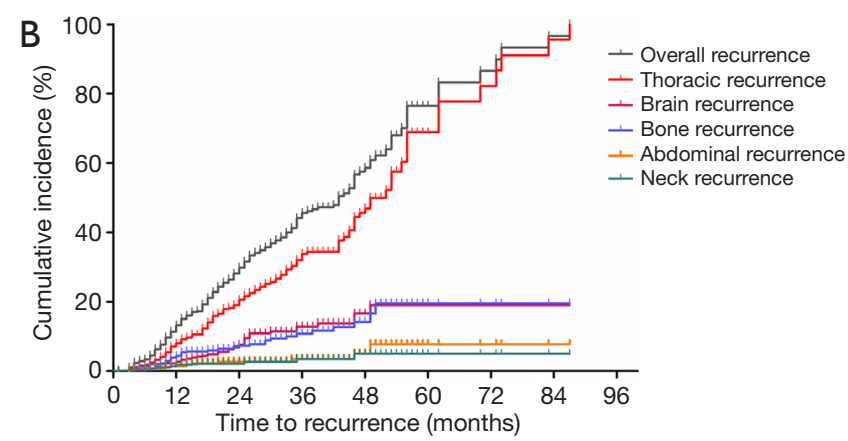

Figure 1 Patterns of initial recurrence in EGFR-mutant NSCLC after curative resection. (A) Pie chart demonstrating the distribution of initial recurrence site; (B) cumulative incidence of overall and site-specific recurrence. EGFR, epidermal growth factor receptor; NSCLC, non-small cell lung cancer.
14 (7.1\%) had abdominal recurrence, 13 (6.6\%) had neck recurrence and $42(21.3 \%)$ had more than one site of initial recurrence. The cumulative incidence of overall and sitespecific recurrence was depicted in Figure 1B. In this figure, patients who developed their initial recurrence in the thoracic region, with or without simultaneous recurrence in other regions, were counted as "thoracic recurrence". This was also the case in other types of recurrence. Of note, recurrence at the resection margin or the anastomosis was observed in 6 patients ( 3 of the patients had distant recurrence simultaneously) and recurrence in the mediastinal or hilar lymph nodes was observed in 28 patients (7 of the patients had distant recurrence simultaneously). Hence, loco-regional recurrence occurred in 34 patients, accounting for $25.0 \%$ of thoracic recurrence.

Specifically, among patients with N2-positive EGFRmutant NSCLC ( $\mathrm{n}=91), 62$ (68.1\%) experienced disease recurrence by the time of data cut-off and the frequencies of thoracic recurrence, brain recurrence, bone recurrence, abdominal recurrence and neck recurrence, were $58.1 \%$, $22.6 \%, 24.2 \%, 12.9 \%$ and $14.5 \%$, respectively. Of note, among patients with $\mathrm{N} 2$-positive disease, initial relapse in the mediastinal or hilar lymph nodes occurred in 11 cases (3 of the patients had distant recurrence simultaneously) and initial recurrence at the resection margin (also had distant recurrence simultaneously) occurred in 1 case.

\section{Risk factors of postoperative recurrence}

Univariate Cox regression analyses revealed that tumor size, $\mathrm{N}$ stage, tumor histology, tumor differentiation, lymphovascular invasion, visceral pleural invasion, as well as Ki67, TTF1, CK20, CK7, and CK5/6 were significantly associated with overall recurrence. Multivariate Cox regression analyses identified tumor size, $\mathrm{N}$ stage, Ki67 and CK20 as independent predictors of overall recurrence (Table 2). In addition, univariate Cox regression analyses of clinical-pathological factors and IHC markers associated with thoracic, brain, bone, abdominal, and neck recurrences, were illustrated in Table S2. Detailed information on the independent indicators of site-specific recurrences was summarized in Table S3. For each site-specific recurrence, initial relapse at other sites were defined as competing events.

Using distant recurrence as a competing event, univariate competing risk analyses found that tumor size, $\mathrm{N}$ stage, tumor histology, lymphovascular invasion, visceral pleural invasion, and CK5/6 were significantly associated with 
Table 2 Univariate and multivariate analyses of clinical-pathological factors and IHC markers associated with overall recurrence

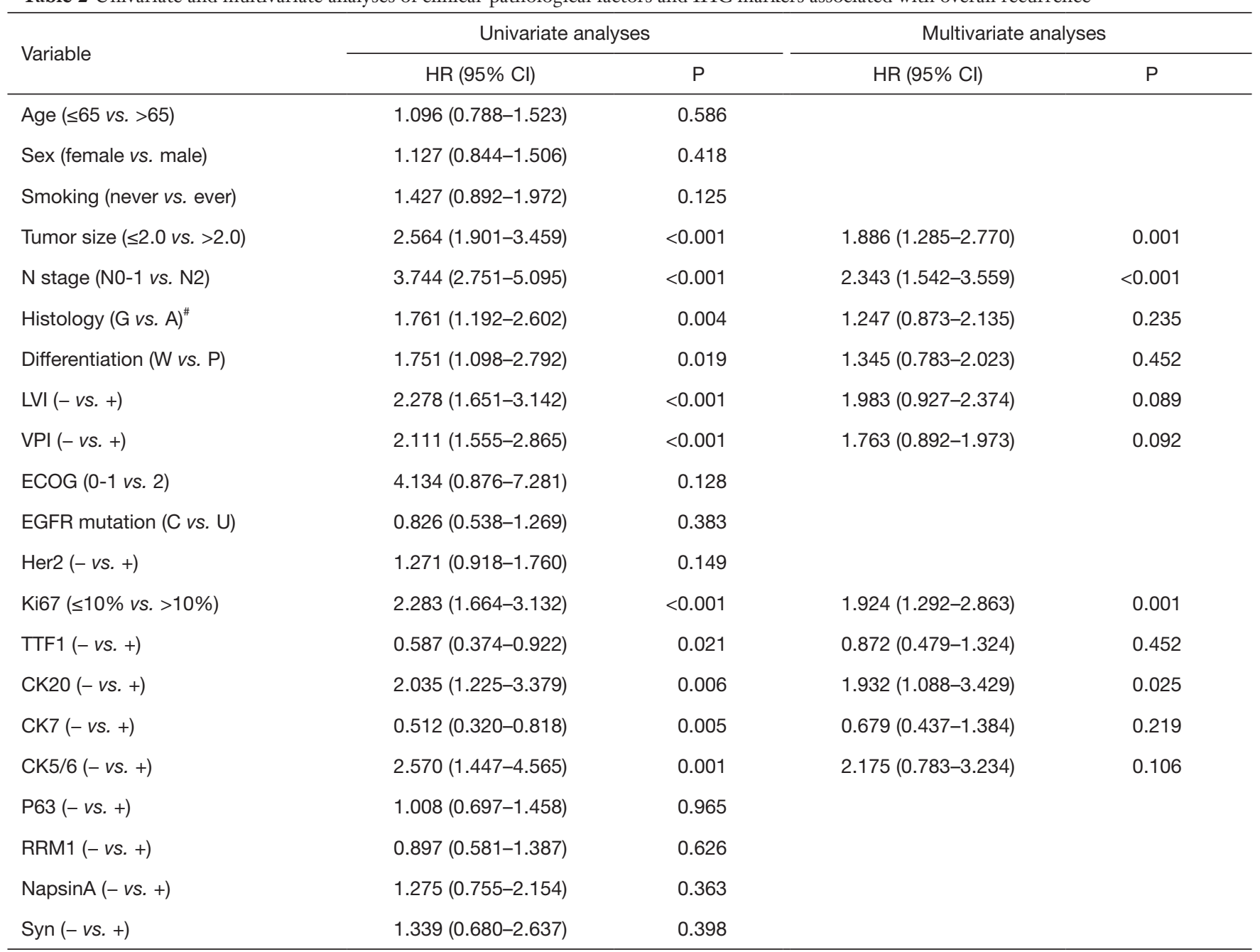

\#, micropapillary subtype of invasive adenocarcinoma, solid subtype of invasive adenocarcinoma and adenosquamous lung carcinomas were defined as "aggressive" (A) tumor histology, while the others were defined as "general" (G). EGFR, epidermal growth factor receptor; $\mathrm{HR}$, hazard ratios; $\mathrm{Cl}$, confidence intervals; LVI, lymphovascular invasion; VPI, visceral pleural invasion; ECOG, the Eastern Corporative Oncology Group; W, well/moderate; P, poor; C, common; U, uncommon.

loco-regional recurrence. Multivariate competing risk analyses revealed that $\mathrm{N}$ stage, lymphovascular invasion and $\mathrm{CK} 5 / 6$ were independent predictors of loco-regional recurrence (Table 3).

\section{Nomogram development and internally validation}

A nomogram based on the four independent indicators of overall recurrence was developed to calculate the 1-, 2-, and 3-year RFS probability (Figure 2). N stage contributed most in the nomogram, followed by CK20 expression, Ki67 index, and tumor size. The concordance index (C-index) of the RFS prediction was 0.754 (95\% CI, 0.685 to 0.791$)$, representing moderate discriminative power of this predictive model. The bias-corrected C-index was 0.723 (95\% CI, 0.675 to 0.771 ), and the extent of "over-fitting" was small $(0.8 \%)$. Calibration curves (Figure 3) depicted good agreement between the predicted 1-, 2-, and 3-year recurrence-free survival and the actual observation from Kaplan-Meier estimates.

\section{Prognostic significance of adjuvant radiotherapy}

Adjuvant radiotherapy is repeated proposed for patients with N2-positive NSCLC $(5,6)$, but its clinical value has 
Table 3 Competing risk analyses of clinical-pathological factors and IHC markers associated with loco-regional recurrence

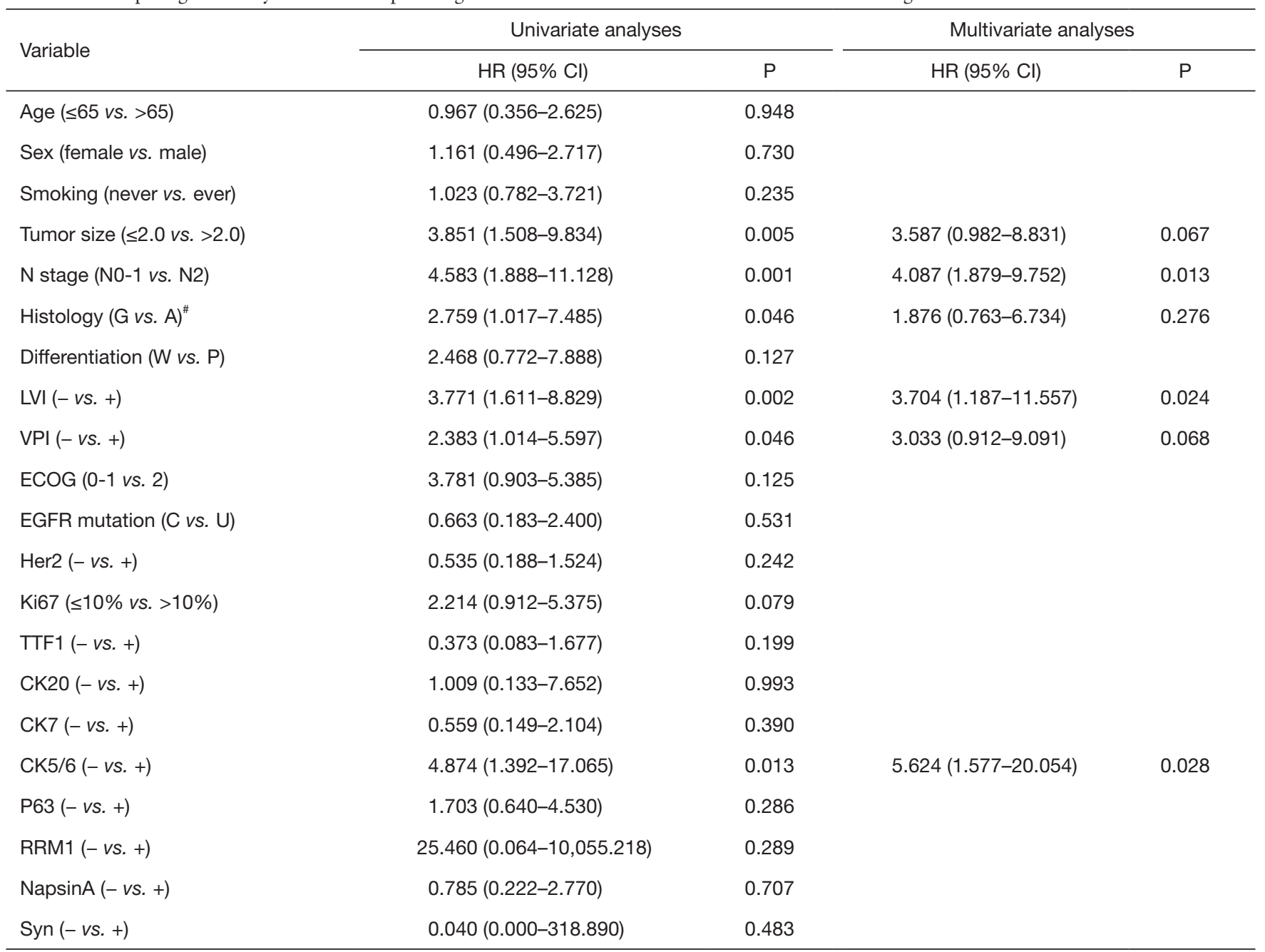

", micropapillary subtype of invasive adenocarcinoma, solid subtype of invasive adenocarcinoma and adenosquamous lung carcinomas were defined as "aggressive" (A) tumor histology, while the others were defined as "general" (G). EGFR, epidermal growth factor receptor; $\mathrm{HR}$, hazard ratios; $\mathrm{Cl}$, confidence intervals; LVI, lymphovascular invasion; VPI, visceral pleural invasion; ECOG, the Eastern Corporative Oncology Group; W, well/moderate; P, poor; C, common; U, uncommon.

not been explored in EGFR-mutant NSCLC. In our study, 28 of the $91 \mathrm{~N} 2$-positive patients received adjuvant radiotherapy, the baseline disease characteristics of those who received adjuvant radiotherapy and those who did not, were generally balanced (Table S4). Adjuvant radiotherapy tended to prolong DFS (Figure $4 A$ ), but failed to improve OS (Figure 4B), among patients with $\mathrm{N} 2$-positive disease. Furthermore, among $61 \mathrm{~N} 2$-positive patients with LVI and/or CK5/6 expression, 15 patients received adjuvant radiotherapy. Among this subgroup of N2-positive patients, adjuvant radiotherapy significantly prolonged DFS $(\mathrm{P}=0.041)$, but again failed to improve $\mathrm{OS}(\mathrm{P}=0.138)$.

\section{Discussion}

Prior investigations have already established a number of clinical-pathological predictive models for evaluating postoperative recurrence risk in NSCLC $(27,28)$, but few study has focused on the patterns and risks of postoperative recurrence from curative resected EGFR-positive NSCLC (29). In addition, recent discoveries have broken new ground in the prognostic significances of IHC biomarkers for survival prediction and risk stratification $(17,30)$. In the current study, we selected ten routine IHC markers and investigated their association with postoperative recurrence in EGFRpositive NSCLC. To the best of our knowledge, this is the 


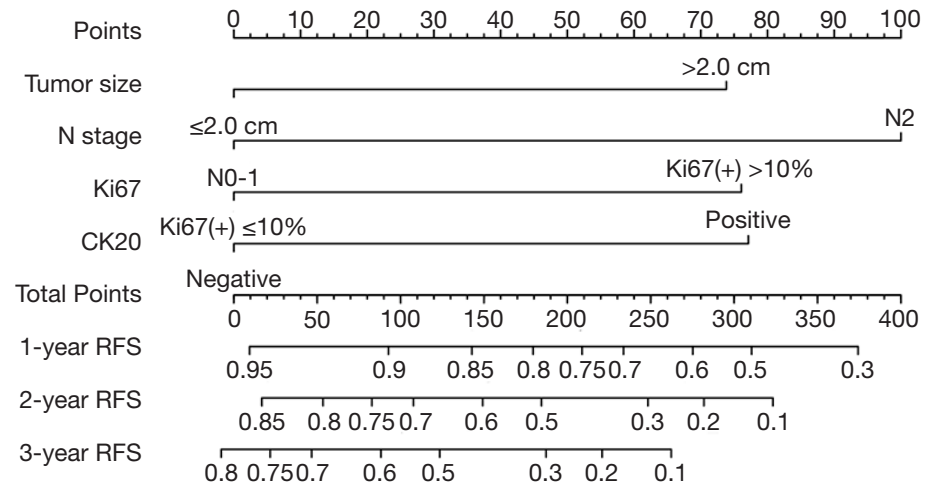

Figure 2 Postoperative nomogram predicting 1-, 2-, 3-year RFS probability. To predict the recurrence risk, users can obtain the score of each indicator first by drawing a line vertically to the scale labeled "Points". Then, add up the corresponding scores of all the indicators. Finally, identify the position of the total score on the "Total Points" scale and draw a line vertically to the "1-Year RFS Probability", "2-Year RFS Probability" and "3-Year RFS Probability" axes to estimate the probability of RFS. RFS, recurrence-free survival; CK20, cytokeratin 20.
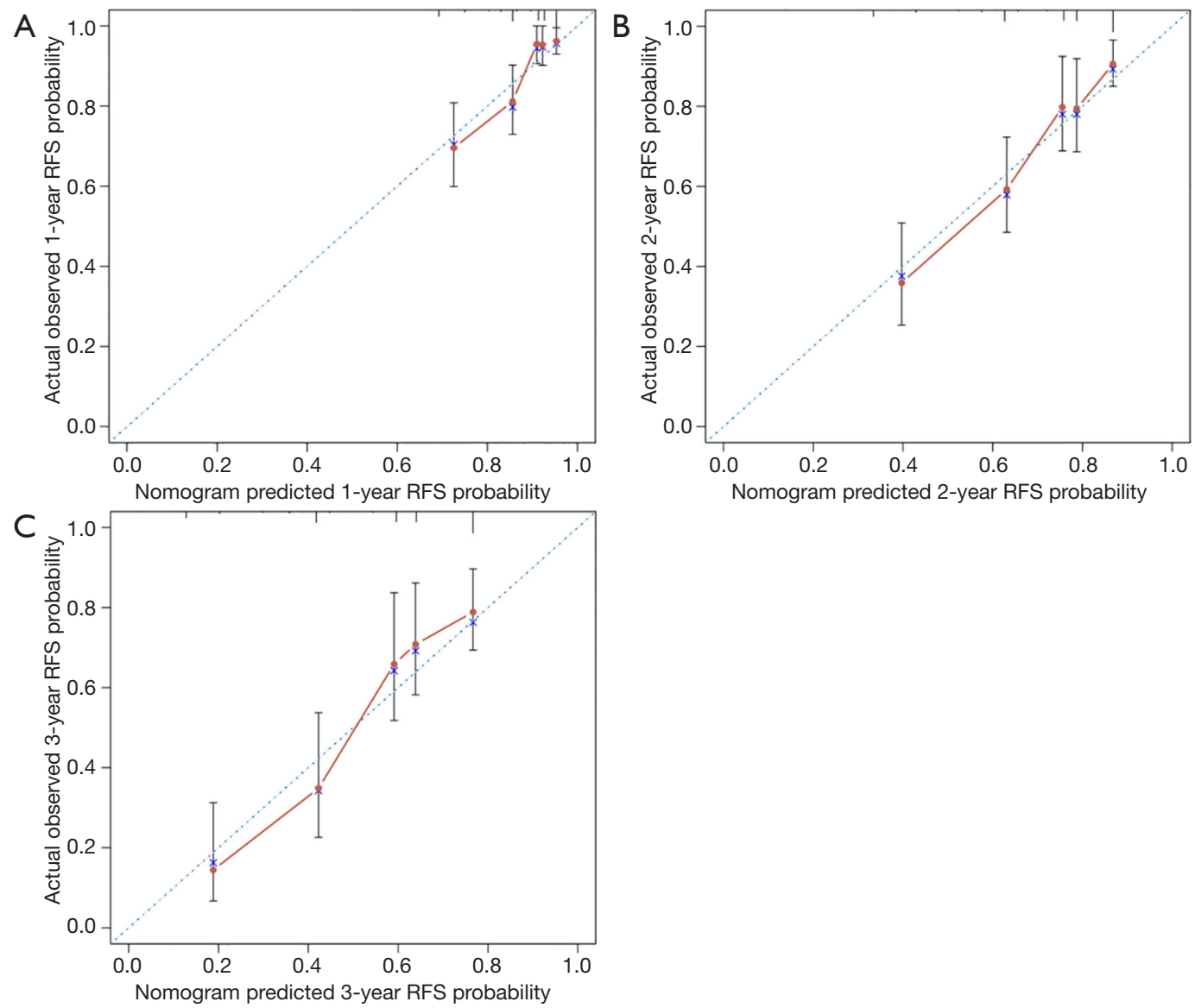

Figure 3 Calibration curves of the nomogram. (A) Comparing nomogram-predicted and actual observed 1-year RFS probability; (B) comparing nomogram-predicted and actual observed 2-year RFS probability; (C) comparing nomogram-predicted and actual observed 3-year RFS probability. The RFS probability predicted by the nomogram is plotted on the X-axis. The actual observed RFS probability is plotted on the Y-axis. The dashed line indicates the ideal concordance of this predictive model. NSCLC, non-small cell lung cancer; EGFR, epidermal growth factor receptor; RFS, recurrence-free survival. 

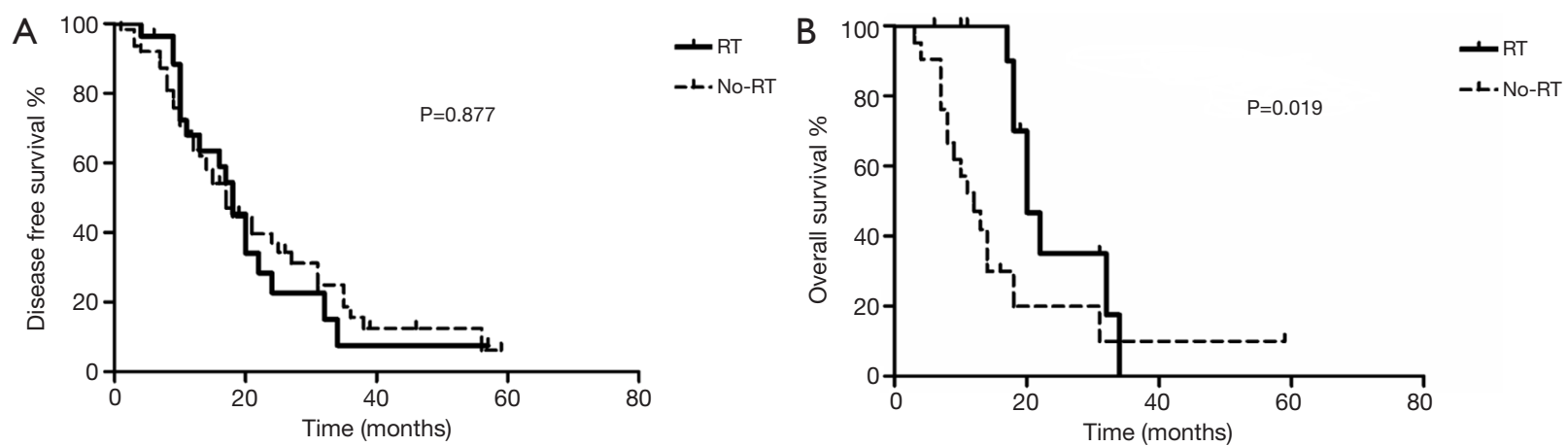

Figure 4 Prognostic significance of adjuvant radiotherapy in EGFR-mutant NSCLC. Kaplan-Meier survival curve stratified by the status of adjuvant radiotherapy among patients with N2-positive disease. (A) disease free survival; (B) overall survival. RT, patients who received adjuvant radiotherapy; No-RT, patients who did not receive adjuvant radiotherapy.

first study incorporating common clinical-pathological parameters and a large panel of routine IHC markers to predict overall, site-specific and loco-regional recurrence, which may help to identify potential candidate of adjuvant therapy in EGFR-mutant NSCLC.

As a convenient tool for diagnosis and classification of lung cancer, IHC analyses of a broad panel of biomarkers were routinely conducted after surgical resection, but their prognostic values have not been fully understood. High level of TTF1 expression was identified as an independent predictive factor of improved prognosis in lung adenocarcinomas (31). The Ki67 index is of great importance for the evaluation of cell proliferation. The combination of Ki67 expression and KRAS mutation status showed an excellent performance in postoperative recurrence prediction for stage I lung adenocarcinoma patients (32). In the current study, we focused on the prognostic significance of an extensive array of routinely used IHC markers and four IHC biomarkers, including Ki67, CK20, CK5/6, and Syn, were finally identified as the independent indicators of overall recurrence and sitespecific recurrence. Syn is a biomarker of neuroendocrine differentiation and highly metastatic clinical behavior of NSCLC with neuroendocrine differentiation has been noted (33). Conversely, other studies found no significant correlation between neuroendocrine markers and the prognosis of NSCLC (34). In our study, Syn was identified as an independent indicator of brain recurrence. Cytokeratins (CKs) are cytoskeletal proteins located in the epithelial cells and previous studies have mainly focused on the their diagnostic roles in the classification of NSCLC and the identification of the origin of tumors with unknown primary $(35,36)$. However, CKs might also act as potential prognosis factors. Basal CKs including CK5/6 and CK14 have been shown to be associated with worse prognosis in lymph node-negative triple-negative breast cancer (37). CK20 expression has been reported to be independent indicators of RFS in pT1 urothelial bladder cancer (30). In the current study, CK5/6 and CK20 were identified to independently associated with thorax recurrence and brain recurrence, respectively. Furthermore, CK20 and Ki67 were both incorporated into the nomogram for predicting overall RFS in EGFR-mutant NSCLC patients. The advantage of our nomogram lies in its clinical utility as an easy-to-use tool of risk prediction. All the clinical-pathological variables and IHC markers included in this nomogram were routinely evaluated after surgical resection. Therefore, physicians can directly obtain the information of each variable, which facilitates the process of decision-making.

One strength of the present study is enrolling only patients receiving standard adjuvant chemotherapy. As shown in Table S1, 29 (4.7\%) patients, including those with stage II-III NSCLC but not receiving adjuvant chemotherapy and those receiving adjuvant EGFR TKIs instead of adjuvant chemotherapy, were excluded. There were several rationales to do this. Firstly, adjuvant platinum-based chemotherapy could significant decrease the risk of postoperative recurrence and alter the patterns of initial relapse among stage II-III NSCLC $(4,38)$. Since this has been widely accepted as the standard of care, patients with stage II-III NSCLC but didn't receive adjuvant chemotherapy were excluded. Secondly, although adjuvant EGFR TKIs were shown to improve DFS compared with adjuvant chemotherapy, concerns remain that EGFR TKIs could not increase the proportion of patients achieving a cure and thus could not significantly 
prolong OS $(39,40)$. However, it has been reported that the patterns of postoperative recurrence between patients receiving adjuvant EGFR TKIs and those receiving adjuvant chemotherapy, are dramatically different (29). In order to rule out the possible confounding factors, patients receiving adjuvant EGFR TKIs were excluded. In fact, the percentage of patients receiving adjuvant EGFR TKIs in our cancer center from January 2007 to December 2017, was quite low $(<3 \%)$.

On the other hand, patients receiving adjuvant radiotherapy and those who did not, were both allowed to be included. Previous studies revealed that postoperative radiation therapy imparted a survival benefit in N2-positive NSCLC $(5,41)$. However, investigations examining the clinical value of adjuvant radiotherapy in $\mathrm{N} 2$-positive EGFR-mutant NSCLC, are seldom reported. In our study, $\mathrm{N}$ stage, lymphovascular invasion and CK5/6 were revealed to be independent indicators of loco-regional recurrence, and adjuvant radiotherapy was found to have a trend to improve DFS in N2-positive patients. As the survival curve separated markedly from the early beginning, it was highly possible that adjuvant radiotherapy could significantly prolong DFS in N2-positive EGFR-mutant NSCLC, if the sample size was larger. Nevertheless, adjuvant radiotherapy failed to improve OS in our study, even in the subgroup of patients with extra risk factors of loco-regional recurrence. Among unselected N2-positive NSCLC, adjuvant radiotherapy was repeated shown to prolong OS in retrospective and population-based studies $(5,6,41)$, but data from prospective randomized clinical trials was limited and conflicting (42). EGFR-mutant NSCLC has a indolent biological behavior and is sensitive to EGFR TKIs after disease progression, and thus patients with EGFR-mutant NSCLC generally have a quite long OS allowing various kinds of concurrent, consolidative or salvage radiotherapy to be administered after tumor recurrence $(38,43,44)$, making it hard for adjuvant radiotherapy to have a significant OS benefit.

Our study also has several limitations. First, as a retrospective study, postoperative follow-ups were generally at the discretion of the treating physicians and were not unified. And thus, patients with asymptomatic recurrence may be underestimated, especially for those with brain and/ or bone metastases. Second, we only performed internal validation for the constructed nomogram. Since the biascorrected C-index displayed moderate discriminative power and the extent of "over-fitting" was small during internal validation, external validation of the nomogram was highly warranted. Lastly, as certain selection bias may exist in our study, the prognostic significance of adjuvant radiotherapy needs to be interpreted with caution. However, as shown in Table S4, the baseline disease characteristics of patients who received adjuvant radiotherapy and those who did not, were generally balanced.

\section{Acknowledgments}

We thank James P. Mahaffey, PhD, from Liwen Bianji, Edanz Editing China (www.liwenbianji.cn/ac), for editing the English text of a draft of this manuscript.

Funding: This project was supported by Shanghai Pujiang Program (No. 15PJD008 to Z Zhu), the National Natural Science Foundation of China (No. 81572963 to Z Zhu) and funding provided by the Shanghai Science and Technology Committee (No. 19411965900 to Z Zhu).

\section{Footnote}

Conflicts of Interest: The authors have no conflicts of interest to declare.

Ethical Statement: The authors are accountable for all aspects of the work in ensuring that questions related to the accuracy or integrity of any part of the work are appropriately investigated and resolved. The institutional review board of Fudan University Shanghai Cancer Center approved the study (IRB\#090977-1).

\section{References}

1. Owonikoko TK, Ragin CC, Belani CP, et al. Lung cancer in elderly patients: an analysis of the surveillance, epidemiology, and end results database. J Clin Oncol 2007;25:5570-7.

2. Donington JS, Kim YT, Tong B, et al. Progress in the Management of Early-Stage Non-Small Cell Lung Cancer in 2017. J Thorac Oncol 2018;13:767-78.

3. Sugimura H, Nichols FC, Yang P, et al. Survival after recurrent nonsmall-cell lung cancer after complete pulmonary resection. Ann Thorac Surg 2007;83:409-17; discussioin 417-8.

4. Burdett S, Pignon JP, Tierney J, et al. Adjuvant chemotherapy for resected early-stage non-small cell lung cancer. Cochrane Database Syst Rev 2015;(3):CD011430.

5. Mikell JL, Gillespie TW, Hall WA, et al. Postoperative radiotherapy is associated with better survival in non-small 
cell lung cancer with involved N2 lymph nodes: results of an analysis of the National Cancer Data Base. J Thorac Oncol 2015;10:462-71.

6. Herskovic A, Mauer E, Christos P, et al. Role of Postoperative Radiotherapy in Pathologic Stage IIIA (N2) Non-Small Cell Lung Cancer in a Prospective Nationwide Oncology Outcomes Database. J Thorac Oncol 2017;12:302-13.

7. Hirsch FR, Bunn PA Jr. EGFR testing in lung cancer is ready for prime time. Lancet Oncol 2009;10:432-3.

8. Pirker R, Buder A, Filipits M. Osimertinib in advanced EGFR T790M-positive non-small-cell lung cancer: the clinical impact of AURA3. Transl Cancer Res 2017;6:S265-9.

9. Yuan Y, Huang Q, Gu C, et al. Disease-free survival improved by use of adjuvant EGFR tyrosine kinase inhibitors in resectable non-small cell lung cancer: an updated meta-analysis. J Thorac Dis 2017;9:5314-21.

10. Yue D, Xu S, Wang Q, et al. Erlotinib versus vinorelbine plus cisplatin as adjuvant therapy in Chinese patients with stage IIIA EGFR mutation-positive non-small-cell lung cancer (EVAN): a randomised, open-label, phase 2 trial. Lancet Respir Med 2018;6:863-73.

11. Pennell NA, Neal JW, Chaft JE, et al. SELECT: A Phase II Trial of Adjuvant Erlotinib in Patients With Resected Epidermal Growth Factor Receptor-Mutant Non-SmallCell Lung Cancer. J Clin Oncol 2019;37:97-104.

12. Zhong WZ, Wang Q, Mao WM, et al. Gefitinib versus vinorelbine plus cisplatin as adjuvant treatment for stage II-IIIA (N1-N2) EGFR-mutant NSCLC (ADJUVANT/ CTONG1104): a randomised, open-label, phase 3 study. Lancet Oncol 2018;19:139-48.

13. Fang $S$, Wang Z. EGFR mutations as a prognostic and predictive marker in non-small-cell lung cancer. Drug Des Devel Ther 2014;8:1595-611.

14. VanderLaan PA, Rangachari D, Mockus SM, et al. Mutations in TP53, PIK3CA, PTEN and other genes in EGFR mutated lung cancers: Correlation with clinical outcomes. Lung Cancer 2017;106:17-21.

15. Blakely CM, Watkins TBK, $W u$ W, et al. Evolution and clinical impact of co-occurring genetic alterations in advanced-stage EGFR-mutant lung cancers. Nat Genet 2017;49:1693-704.

16. Ong CA, Shapiro J, Nason KS, et al. Three-gene immunohistochemical panel adds to clinical staging algorithms to predict prognosis for patients with esophageal adenocarcinoma. J Clin Oncol 2013;31:1576-82.

17. Meng J, Zhang J, Xiu Y, et al. Prognostic value of an immunohistochemical signature in patients with esophageal squamous cell carcinoma undergoing radical esophagectomy. Mol Oncol 2018;12:196-207.

18. Sikic D, Keck B, Wach S, et al. Immunohistochemiocal subtyping using CK20 and CK5 can identify urothelial carcinomas of the upper urinary tract with a poor prognosis. PLoS One 2017;12:e0179602.

19. Inwald EC, Koller M, Klinkhammer-Schalke M, et al. 4-IHC classification of breast cancer subtypes in a large cohort of a clinical cancer registry: use in clinical routine for therapeutic decisions and its effect on survival. Breast Cancer Res Treat 2015;153:647-58.

20. Osmani L, Askin F, Gabrielson E, et al. Current WHO guidelines and the critical role of immunohistochemical markers in the subclassification of non-small cell lung carcinoma (NSCLC): Moving from targeted therapy to immunotherapy. Semin Cancer Biol 2018;52:103-9.

21. Rami-Porta R, Wittekind C, Goldstraw P, et al. Complete resection in lung cancer surgery: proposed definition. Lung Cancer 2005;49:25-33.

22. NCCN Clinical Practice Guidelines in Oncology: Nonsmall cell lung cancer. Version 5.2019-June 7, 2019. Available online: https://www.Nccn.Org/professionals. Accessed 28 July, 2019.

23. Detterbeck FC, Boffa DJ, Kim AW, et al. The Eighth Edition Lung Cancer Stage Classification. Chest 2017;151:193-203.

24. Travis WD, Brambilla E, Nicholson AG, et al. The 2015 World Health Organization Classification of Lung Tumors: Impact of Genetic, Clinical and Radiologic Advances Since the 2004 Classification. J Thorac Oncol 2015;10:1243-60.

25. Travis WD, Brambilla E, Noguchi M, et al. International association for the study of lung cancer/american thoracic society/european respiratory society international multidisciplinary classification of lung adenocarcinoma. J Thorac Oncol 2011;6:244-85.

26. Harrell FE, Jr., Lee KL, Mark DB. Multivariable prognostic models: issues in developing models, evaluating assumptions and adequacy, and measuring and reducing errors. Stat Med 1996;15:361-87.

27. Chen H, Sui X, Yang F, et al. Nomograms for predicting recurrence and survival of invasive pathological stage IA non-small cell lung cancer treated by video assisted thoracoscopic surgery lobectomy. J Thorac Dis 2017;9:1046-53.

28. Zhang Y, Zheng D, Xie J, et al. Development and Validation of Web-Based Nomograms to Precisely Predict 
Conditional Risk of Site-Specific Recurrence for Patients With Completely Resected Non-small Cell Lung Cancer: A Multiinstitutional Study. Chest 2018;154:501-11.

29. Xu ST, Xi JJ, Zhong WZ, et al. The Unique SpatialTemporal Treatment Failure Patterns of Adjuvant Gefitinib Therapy: A Post Hoc Analysis of the ADJUVANT Trial (CTONG 1104). J Thorac Oncol 2019;14:503-12.

30. Bertz S, Otto W, Denzinger S, et al. Combination of CK20 and Ki-67 immunostaining analysis predicts recurrence, progression, and cancer-specific survival in pT1 urothelial bladder cancer. Eur Urol 2014;65:218-26.

31. Perner S, Wagner PL, Soltermann A, et al. TTF1 expression in non-small cell lung carcinoma: association with TTF1 gene amplification and improved survival. J Pathol 2009;217:65-72.

32. Woo T, Okudela K, Yazawa T, et al. Prognostic value of KRAS mutations and $\mathrm{Ki}-67$ expression in stage I lung adenocarcinomas. Lung Cancer 2009;65:355-62.

33. Sundaresan V, Reeve JG, Stenning S, et al. Neuroendocrine differentiation and clinical behaviour in non-small cell lung tumours. British journal of cancer 1991;64:333-8.

34. Graziano SL, Tatum AH, Newman NB, et al. The prognostic significance of neuroendocrine markers and carcinoembryonic antigen in patients with resected stage I and II non-small cell lung cancer. Cancer Res 1994;54:2908-13.

35. Chen Y, Cui T, Yang L, et al. The diagnostic value of cytokeratin 5/6,14, 17, and 18 expression in human nonsmall cell lung cancer. Oncology 2011;80:333-40.

36. Montezuma D, Azevedo R, Lopes P, et al. A panel of four immunohistochemical markers (CK7, CK20, TTF-1, and p63) allows accurate diagnosis of primary and metastatic lung carcinoma on biopsy specimens. Virchows Arch 2013;463:749-54.

37. Rakha EA, El-Sayed ME, Green AR, et al. Prognostic markers in triple-negative breast cancer. Cancer 2007;109:25-32.

38. Wu F, Liu X, Ma JA, et al. Adjuvant therapy for resected EGFR-mutant non-small-cell lung cancer. Lancet Oncol 2018;19:e124.

39. Ma JA, Jiang S, Hu C, et al. Adjuvant therapy for resected EGFR-mutant non-small-cell lung cancer. Lancet Oncol 2018;19:e125.

40. Ng TL, Camidge DR. Lung cancer's real adjuvant EGFR targeted therapy questions. Lancet Oncol 2018;19:15-7.

41. Robinson CG, Patel AP, Bradley JD, et al. Postoperative radiotherapy for pathologic N2 non-small-cell lung cancer treated with adjuvant chemotherapy: a review of the National Cancer Data Base. J Clin Oncol 2015;33:870-6.

42. Sun JM, Noh JM, Oh D, et al. Randomized Phase II Trial Comparing Chemoradiotherapy with Chemotherapy for Completely Resected Unsuspected N2-Positive Non-Small Cell Lung Cancer. J Thorac Oncol 2017;12:1806-13.

43. Zheng L, Wang Y, Xu Z, et al. Concurrent EGFR-TKI and Thoracic Radiotherapy as First-Line Treatment for Stage IV Non-Small Cell Lung Cancer Harboring EGFR Active Mutations. Oncologist 2019;24:1031-e612.

44. Xu Q, Zhou F, Liu H, et al. Consolidative Local Ablative Therapy Improves the Survival of Patients With Synchronous Oligometastatic NSCLC Harboring EGFR Activating Mutation Treated With First-Line EGFRTKIs. J Thorac Oncol 2018;13:1383-92.
Cite this article as: Ni J, Guo T, Li Y, Yang X, Li Y, Zou L, Chu L, Chu X, Li S, Ye L, Zhang Y, Zhu Z. Patterns and risks of postoperative recurrence in completely resected EGFRmutant non-small cell lung cancer: prognostic significance of routine immunohistochemical markers. Transl Lung Cancer Res 2019;8(6):967-978. doi: 10.21037/tlcr.2019.12.02 


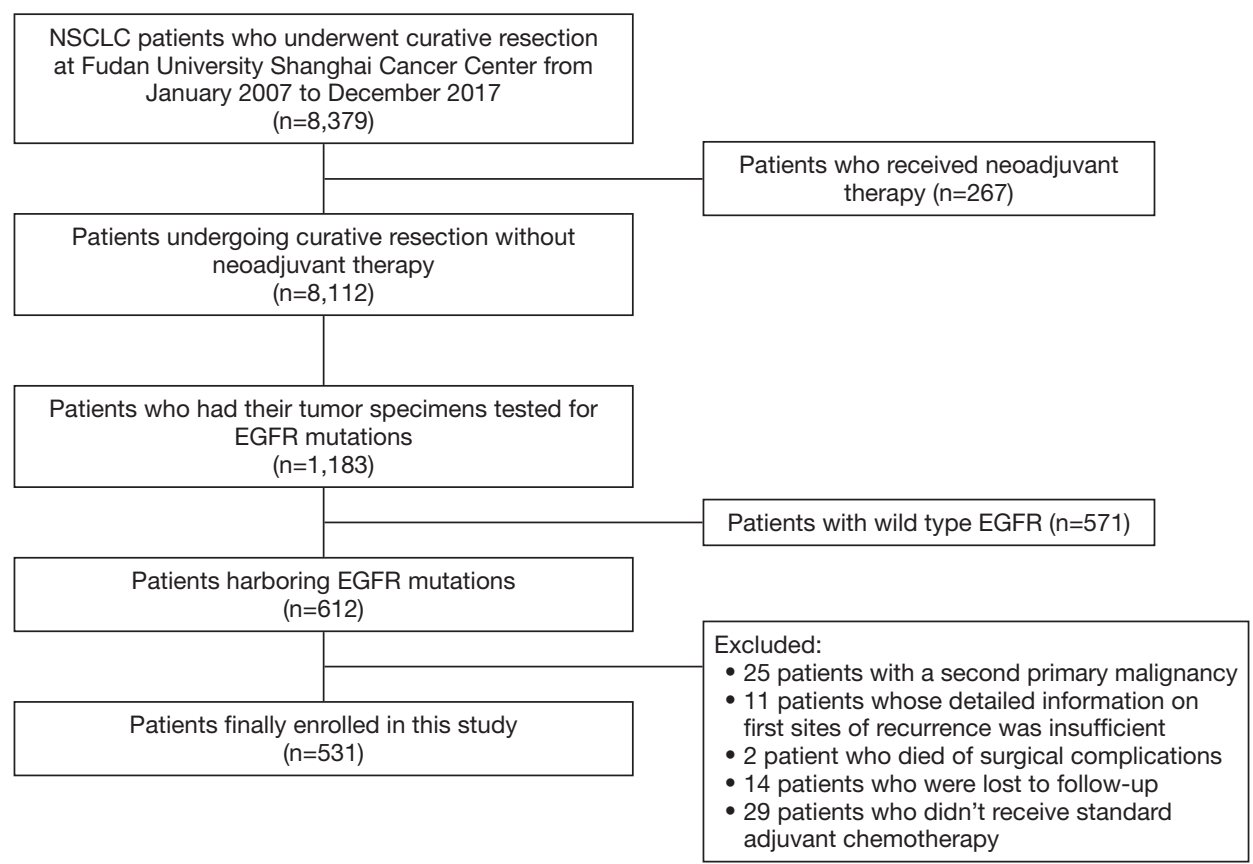

Figure S1 Flowchart of patient enrollment. NSCLC, non-small cell lung cancer; EGFR, epidermal growth factor receptor.

Table S1 Baseline characteristics of NSCLC patients who underwent curative resection and who had their EGFR mutation tested after curative resection from January 2007 to December 2017

\begin{tabular}{lcc}
\hline Variable & The whole population $(\mathrm{n}=8,379)$ & EGFR tested $(\mathrm{n}=1,183)$ \\
\hline Age (years) & & $\mathrm{P}$ \\
$\leq 65$ & $6,032(72.0 \%)$ & $340(71.0 \%)$ \\
$>65$ & $2,347(28.0 \%)$ & $343(29.0 \%)$ \\
Sex & & $746(63.1 \%)$ \\
Female & $5,274(62.9 \%)$ & $437(36.9 \%)$ \\
Male & $3,105(37.1 \%)$ & 0.938 \\
Pathologic TNM stage & & $844(71.3 \%)$ \\
I & $6,123(73.1 \%)$ & $189(16.0 \%)$ \\
II & $1,347(16.1 \%)$ & $150(12.7 \%)$ \\
III & $909(10.8 \%)$ & 0.168 \\
Histology & & $279(23.6 \%)$ \\
Squamous carcinoma & $2,053(24.5 \%)$ & $904(76.4 \%)$ \\
Non-squamous carcinoma & $6,326(75.5 \%)$ & 0.491 \\
\hline
\end{tabular}

NSCLC, non-small cell lung cancer; EGFR, epidermal growth factor receptor. 


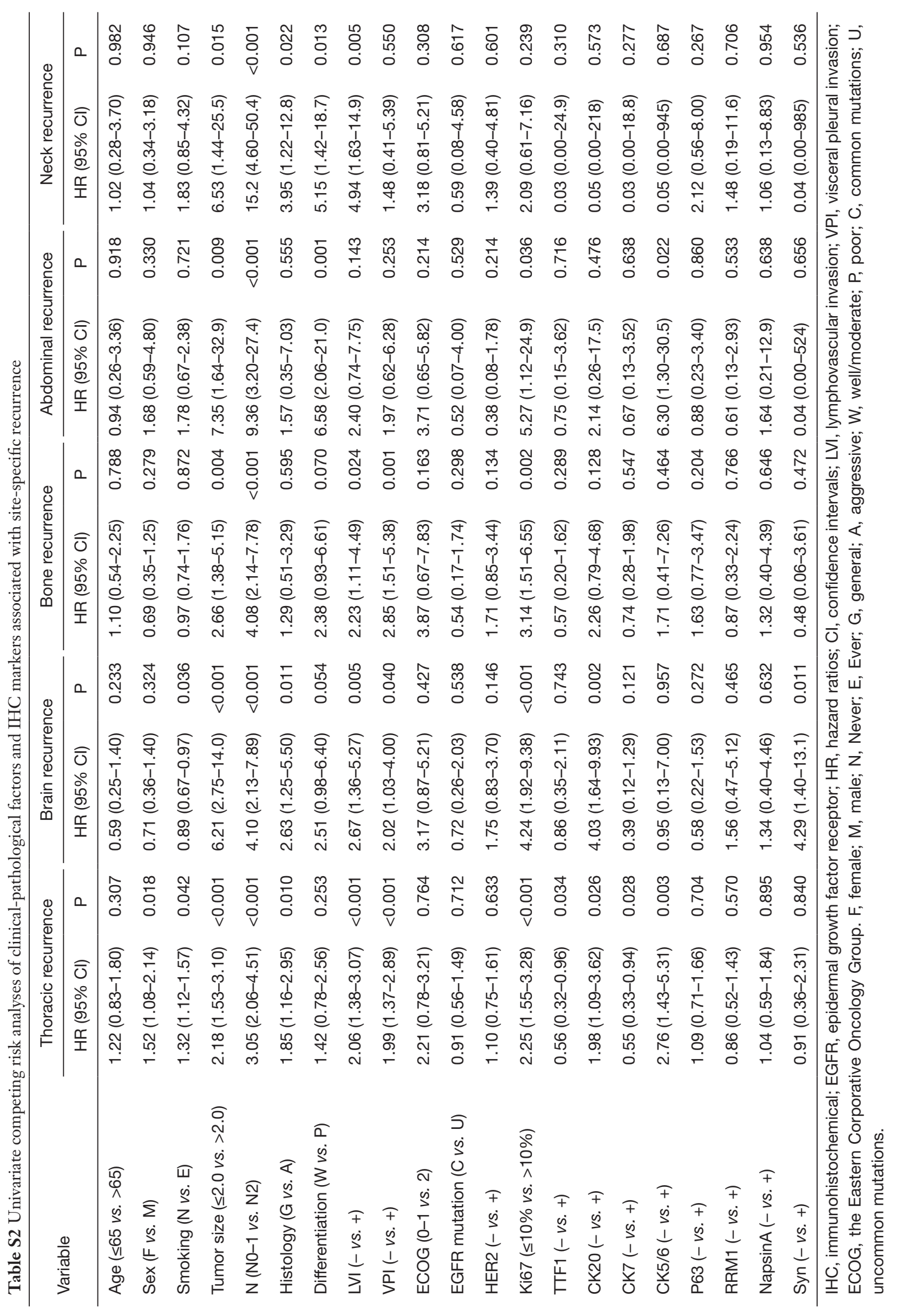


Table S3 Multivariate competing risk analyses of independent indicators of site-specific recurrence

\begin{tabular}{|c|c|c|c|}
\hline Variable & $\mathrm{HR}$ & $95 \% \mathrm{Cl}$ & $\mathrm{P}$ \\
\hline \multicolumn{4}{|l|}{ Thoracic recurrence } \\
\hline Tumor size $(\leq 2.0$ vs. $>2.0 \mathrm{~cm})$ & 1.167 & $1.147-2.648$ & 0.009 \\
\hline Sex (female vs. male) & 1.671 & $1.103-2.517$ & 0.036 \\
\hline N stage (N0-1 vs. N2) & 1.643 & $1.128-2.837$ & 0.028 \\
\hline LVI (absence vs. presence) & 1.997 & $0.905-2.813$ & 0.245 \\
\hline VPI (absence vs. presence) & 1.649 & $0.782-2.422$ & 0.337 \\
\hline TTF1 (- vs. +) & 0.721 & $0.507-1.371$ & 0.573 \\
\hline CK20 (negative vs. positive) & 1.878 & $0.907-3.872$ & 0.224 \\
\hline CK7 (- vs. +$)$ & 0.892 & $0.407-2.034$ & 0.417 \\
\hline \multicolumn{4}{|l|}{ Brain recurrence } \\
\hline Tumor size $(\leq 2.0 \mathrm{vs} .>2.0 \mathrm{~cm})$ & 9.273 & $1.374-72.12$ & 0.031 \\
\hline N stage (N0-1 vs. N2) & 3.721 & $1.172-11.97$ & 0.038 \\
\hline CK20 (negative vs. positive) & 4.271 & $1.452-13.18$ & 0.017 \\
\hline Syn (negative vs. positive) & 4.378 & $1.429-14.95$ & 0.015 \\
\hline Histology (general vs. aggressive) & 1.973 & $0.962-3.973$ & 0.183 \\
\hline LVI (absence vs. presence) & 2.436 & $0.783-3.263$ & 0.318 \\
\hline VPI (absence vs. presence) & 1.887 & $0.621-2.872$ & 0.445 \\
\hline Ki67 ( $\leq 10 \%$ vs. $>10 \%)$ & 3.863 & $0.918-4.883$ & 0.099 \\
\hline VPI (absence vs. presence) & 2.357 & $0.924-4.872$ & 0.372 \\
\hline \multicolumn{4}{|l|}{ Abdominal recurrence } \\
\hline $\mathrm{N}$ stage (N0-1 vs. N2) & 11.047 & $2.891-42.209$ & $<0.001$ \\
\hline Tumor size $(\leq 2.0$ vs. $>2.0 \mathrm{~cm})$ & 5.236 & $0.783-10.237$ & 0.127 \\
\hline Differentiation (well/moderate vs. poor) & 4.371 & $0.639-18.473$ & 0.378 \\
\hline Ki67 ( $\leq 10 \%$ vs. $>10 \%)$ & 4.872 & $0.579-11.238$ & 0.381 \\
\hline CK5/6 (negative vs. positive) & 5.269 & $0.421-17.215$ & 0.457 \\
\hline \multicolumn{4}{|l|}{ Neck recurrence } \\
\hline $\mathrm{N}$ stage (N0-1 vs. N2) & 15.236 & $4.607-50.390$ & $<0.001$ \\
\hline Tumor size $(\leq 2.0$ vs. $>2.0 \mathrm{~cm})$ & 5.792 & $0.892-10.378$ & 0.218 \\
\hline Differentiation (well/moderate vs. poor) & 4.831 & $0.736-11.861$ & 0.387 \\
\hline Histology (general vs. aggressive) & 2.871 & $0.674-9.763$ & 0.217 \\
\hline LVI (absence vs. presence) & 4.082 & $0.873-8.973$ & 0.327 \\
\hline
\end{tabular}

$\mathrm{HR}$, hazard ratios; Cl, confidence intervals; LVI, lymphovascular invasion; VPI, visceral pleural invasion. 
Table S4 Baseline characteristics of stage III/N2 patients who received adjuvant RT and those who didn't (non-RT)

\begin{tabular}{|c|c|c|c|}
\hline Variable & $\mathrm{RT}$ & Non-RT & $P$ \\
\hline Age (years) & & & 0.163 \\
\hline$\leq 65$ & 25 & 47 & \\
\hline$>65$ & 3 & 16 & \\
\hline Sex & & & 0.547 \\
\hline Female & 15 & 38 & \\
\hline Male & 13 & 25 & \\
\hline Pathologic T stage & & & 0.067 \\
\hline T1 & 11 & 37 & \\
\hline $\mathrm{T} 2$ & 15 & 18 & \\
\hline T3 & 2 & 3 & \\
\hline T4 & 0 & 5 & \\
\hline Histology & & & 0.523 \\
\hline Adenosquamous carcinoma & 1 & 1 & \\
\hline Adenocarcinoma & 27 & 62 & \\
\hline ECOG performance score & & & 0.711 \\
\hline $0-1$ & 26 & 57 & \\
\hline 2 & 2 & 6 & \\
\hline
\end{tabular}

$\mathrm{RT}$, radiotherapy; ECOG, the Eastern Corporative Oncology Group. 